EISSN: 2706-7955 ISSN: 2077-4605

DOI: $10.36632 /$ mejar/2021.10.1.21

Journal homepage: www.curresweb.com

Pages: 342-357

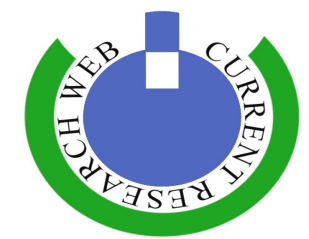

\title{
Evaluate productivity and pest resistance in response to the application of organic fertilizers on Hibiscus sabdariffa L. plants
}

\author{
El-Mogy E.E.A.M. ${ }^{1}$, K. E. Attia ${ }^{1}$, Mona S. Megahed ${ }^{1}$ and M.M.A. Ibrahim ${ }^{2}$ \\ ${ }^{I}$ Medicinal and Aromatic Plants Research Department, Horticulture Research Institute, ARC, Egypt \\ ${ }^{2}$ Piercing-sucking insect Research Department, plant protection Research Institute, ARC. Egypt \\ Received: 20 December 2020 Accepted: 05 March $2021 \quad$ Published: 15 March 2021
}

\begin{abstract}
Two trials were conducted during the two successive seasons of 2017 and 2018 at the Experimental Farm of Horticulture Research Station, in El-Quassassin, Ismailia, Egypt to investigate the effect of effective microorganisms $\left(\mathrm{EM}_{1}\right)$, humic acid, microelements and different doses of NPK fertilizers under different rates of compost on the growth, yield, anthocyanin and NPK contents of Roselle (Hibiscus sabdariffa L.) plants, in addition to investigate its susceptibility to infestation with insect and mite pests. The results showed that, the highest values of plant height, number of branches/plant, number of fruits/plant, fresh weight of fruits/plant, seed yield/plant, dry weight of calyces $(\mathrm{g}) / \mathrm{plant}$, yield of dry calyces $(\mathrm{kg} / \mathrm{fed}$.) and anthocyanins content in dry sepals, were obtained from the treatment of $\left[25 \%\right.$ compost $\left(3 \mathrm{~m}^{3} /\right.$ fed. $)+(75 \%$ NPK $(225 \mathrm{~kg}$ ammonium sulphate $/$ fed. $+225 \mathrm{~kg}$ calcium super phosphate/fed. $+75 \mathrm{~kg}$ potassium sulphate/fed.)], followed by $\left[100 \%\right.$ compost $\left(12 \mathrm{~m}^{3} / \mathrm{fed}\right.$. $)+\mathrm{EM}_{1}$ as 5 liters/fed. (soil adding) + Humic acid as $280 \mathrm{~g} / \mathrm{fed}$. (soil adding) + Microelements as $1 \mathrm{~kg} / \mathrm{fed}$. (soil adding)] and [100\% NPK (300 kg ammonium sulphate/fed. $+300 \mathrm{~kg}$ calcium super phosphate/fed. +100 $\mathrm{kg}$ potassium sulphate/fed.)], without significant differences between them, in most cases, during the two seasons. Regarding the pests associated with Roselle plants, under different treatments, more than one species of insects and insect predators were observed. In regard to the pests of Roselle plants, the all or some tested treatments found infested with more than one species of leafhoppers, three species of aphids, cotton whitefly, onion thrips, green stink bug, cotton seeds bug, spiny boll worm, and the two spotted spider mite. Also, these pests found associated with four species of insect predators. The leafhoppers, Empoasca spp. insects and T. urticea were the dominant piercing-sucking pests infesting Roselle plants throughout the whole season and $O$. hyalinipennis during fruiting period. In the same trend, highly significant differences $(\mathrm{P} \leq 0.01)$ were noticed between the mean numbers of the surveyed pests and associated natural enemies; which were resulted as the variation of chemical, bio and organic fertilizers rates and types treatments. In where, the relatively low mean numbers of investigated pests of $2.00,6.33,2.00,1.33,1.00,15.00,8.33$ individual/sample unite were recorded in the organic fertilizers treatments for Empoasca spp., Aphis gossypii, Bemisia tabaci, Thrips tabaci, Nezara viridula, Oxycarenus hyalinipennis and Tetranychus urticea during the study period, respectively. Also, significant correlation coefficients were obtained between the mean numbers of surveyed pests and each of plant height, number of branches/plant. According to these results, mineral fertilizers could be replaced with compost, EM and Humic acid for producing good yield of healthy Roselle sepals and to avoid bad side effects of agrochemicals with the aim to get safe foods.
\end{abstract}

Keywords: Roselle, compost, effective microorganisms ( $\left.\mathrm{EM}_{1}\right)$, Humic acid, Microelements, Calyces, Anthocyanins, Roselle pests and associated natural enemies.

\section{Introduction}

Roselle (Hibiscus sabdariffa L.) plant was cultivated especially for the important commercial part of the plant which is the dried sepals (calyx) surrounding the fruit (capsules). Also, it cultivated for its leaf, fleshy calyx, seed or fiber (Dalziel, 1973). The genus Hibiscus (Fam. Malvaceae) includes more

Corresponding Author: El-Mogy E.E.A.M., Medicinal and Aromatic Plants Research Department, Horticulture Research Institute, ARC, Egypt 
than 300 species of annual or perennial herbs, shrubs or trees (Wang et al., 2012). It is considered to be a native to Asia (India to Malaysia) or Tropical Africa. This plant can grow on a wide range of soil conditions, but for economic production; soil should be well supplied with important fertilizers which can improve sepals yield and their quality.

Tsai et al (2002) indicated that, anthocyanin is the major source of antioxidant capacity in Roselle extract. Anthocyanins are a group of flavonoid derivatives and natural pigments present in the dried calyxes and their color varies with $\mathrm{pH}$.

Decoctions of Roselle have been used traditionally as an anti-hypertensive remedy. Many authors found that, Roselle lowering the systolic blood pressure and diastolic pressure in the patients (Khosravi et al., 2009 and Esmaeili et al., 2014). Moreover, anthocyanin can cause cancer cell apoptosis, especially in HL-60 cells (Chang et al., 2005).

Organic fertilizers can be used to reduce the amount of toxic compounds (such as nitrate) produced by chemical fertilizers; they improve the quality of vegetables and safe for human health (Mahmoud et al., 2009). Compost improves physical, biological and chemical properties of soil (Albiach et al., 2000 and Baziramakenga and Simard, 2001).

In some countries, producing Egyptian Roselle requires a fair trade certificate to prove originality of the product. Well-knowingly, organic farming increases value of different products in agriculture and food trade (Bavec and Bavec, 2006). Many authors used compost to raise the quantity and quality of Roselle. Cassava peel compost (CPC) with NPK fertilizers caused significant increases in yield and quality of calyx compared with NPK alone (Akanbi et al., 2009).

Many reports showed that spraying plants with humic acid resulted in a better plant growth and fruit yield._Azarpour et al (2012) stated that, the effect of humic acid as foliar spraying on eggplant (Solanum melongena L.) at all examined doses $(25$ and $50 \mathrm{mg} / \mathrm{L}$.) had significant differences at $1 \%$ probability level of fruit yield, number of fruits per square meter, number of branches per plant and plant height.

The importance of foliar fertilization with micronutrients, $\mathrm{Fe}, \mathrm{Zn}$ and $\mathrm{Mn}$ can be accounted by its essential role in respiration, their metabolism activation of the enzyme, photosynthesis, chloroplast formation, chlorophyll synthesis and natural hormone biosynthesis (Epstien, 1972 and Mortvedt, et al., 1991). Hussein et al (1989) indicated that, the micronutrients (Fe or Mn at 25 p.p.m.), as foliar sprays on $H$. sabdariffa, stimulated growth and increased yield components. Fe increased the endogenous gibberellin level.

Concerning using macronutrients with organic fertilizer, Maunde and Aliyu (2012) found that, the application of $60 \mathrm{~kg} \mathrm{~N} \mathrm{ha}^{-1}$, and $5 \mathrm{t} \mathrm{FYM} \mathrm{ha}^{-1}$ significantly increased total dry matter yield and height of Roselle. On the other wise, combined application of $120 \mathrm{~kg} \mathrm{~N}$ with $5 \mathrm{t} \mathrm{FYM} \mathrm{ha}{ }^{-1}$ was optimum for total dry matter yield of Roselle.

In Egypt, Roselle plants are subjected to be attacked by many pests species, which cause economic damage throughout vegetative, fruiting periods and seed in both quantity and quality. Piercing sucking pests (aphid's species, leafhoppers, whiteflies, thrips, stink bugs and mites) and spiny boll worm are the most destructive insects on these plants. Mesbah et al, (1982), Ahmad (1990), El Sayed et al, (1990), Ahmed et al (1995), Hammad and Mohsen (2000), Abd El-Moniem and Abd El-Wahab (2006), Afsah (2005). Cotton aphid, Aphis gossypii and leafhopper, Empoasca spp. reported to be the main insect species infesting Roselle plants and causes high percent of losses. On the other side, Roselle fields may harbor beneficial insects, such as predators, parasitoids, which play a noticeable role in controlling the pests and subsequently improving the production of these plants (Rashad, 1976 and 1978; Abd EL-Moniem and Abd EL-Wahab, 2006 and Abd EL-Karim et al., 2011). Also, many entomologists reported that there is strong relationship between the levels of nitrogen fertilization, amino acids and the levels of piercing-sucking pests infestation, which followed by the appearance of their predators and parasitoids. Moreover, different phonologies of Roselle have been reportedly attacked by insects which cause economic damage, attacking the lamina of the young foliage and matured leaves therefore result in reduction of the photosynthetic ability of the crop.

This study aims to evaluate the possibility replace the mineral fertilizers for producing Roselle plant partly or totally by using compost, EM and Humic; and to study the impact of these materials on the plants growth, yield components and pests infestation. 


\section{Materials and Methods}

Two experiments were conducted during two successive seasons (2017 and 2018) at the Experimental Farm of Horticulture Research Station, in El-Quassassin, Ismailia, Egypt to evaluate the possibility to replace the mineral fertilizers $(\mathrm{N}, \mathrm{P}$ and $\mathrm{K}$ ) for producing Roselle plant partly or totally by using different rates of compost, EM and humic acid.

Seeds of Roselle were obtained from Medicinal and Aromatic Plants Department, Horticulture Research Institute, Agriculture Research Center, Giza, Egypt. Seeds were sown in sandy soil on the $3^{\text {rd }}$ May for both seasons. Each treatment was planted in 3 rows, each row was $5 \mathrm{~m}$ long and $0.7 \mathrm{~m}$ wide; making an area of $10.5 \mathrm{~m}^{2}$. Hills were $50 \mathrm{~cm}$ apart; 5 seeds per hill then thinned three weeks later to one plant/hill. Other agricultural practices such as :irrigation and weeding were carried out as recommended by ministry of Agriculture in Egypt.

Fifteen treatments comprising $\mathrm{EM}_{1}$, humic acid, microelements and different doses of NPK fertilizers under different rates of compost were applied in this experiment as follow:

1 - $100 \%$ compost $\left(12 \mathrm{~m}^{3} /\right.$ fed. $)$

2- $100 \%$ compost $+\mathrm{EM}_{1}$ (soil adding)

3- $100 \%$ compost + humic acid (soil adding)

4- $100 \%$ compost + humic acid (foliar spraying)

5- $100 \%$ compost + microelements (soil adding)

6- $100 \%$ compost + microelements (foliar spraying)

7- $100 \%$ compost $+\mathrm{EM}_{1}$ (soil adding) + humic acid (soil adding)

8- $100 \%$ compost $+\mathrm{EM}_{1}$ (soil adding) + microelements (soil adding)

9- $100 \%$ compost + humic acid (foliar spraying) + microelements (foliar spraying)

$10-100 \%$ compost + humic acid (soil adding) + microelements (soil adding)

$11-100 \%$ compost $+\mathrm{EM}_{1}$ (soil adding) + humic acid (soil adding) + microelements (soil adding)

12- $75 \%$ compost $\left(9 \mathrm{~m}^{3} /\right.$ fed. $)+\left[25 \%\right.$ NPK $\left(75 \mathrm{~kg} \mathrm{~N} /\right.$ fed. $+75 \mathrm{~kg} \mathrm{P}_{2} \mathrm{O}_{5} /$ fed. $+25 \mathrm{~kg} \mathrm{~K} 2 \mathrm{O} /$ fed. $\left.)\right]$

13- $50 \%$ compost $\left(6 \mathrm{~m}^{3} /\right.$ fed. $)+\left[50 \% \mathrm{NPK}\left(150 \mathrm{~kg} \mathrm{~N} /\right.\right.$ fed. $+150 \mathrm{~kg} \mathrm{P}_{2} \mathrm{O}_{5} /$ fed. $+50 \mathrm{~kg} \mathrm{~K} 2 \mathrm{O} /$ fed. $\left.)\right]$

14- $25 \%$ compost $\left(3 \mathrm{~m}^{3} /\right.$ fed. $)+\left[75 \% \mathrm{NPK}\left(225 \mathrm{~kg} \mathrm{~N} /\right.\right.$ fed. $+225 \mathrm{~kg} \mathrm{P}_{2} \mathrm{O}_{5} /$ fed. $+75 \mathrm{~kg} \mathrm{~K} 2 \mathrm{O} / \mathrm{fed}$. $\left.)\right]$

15- $100 \%$ NPK (300 kg N/fed. $+300 \mathrm{~kg} \mathrm{P}_{2} \mathrm{O}_{5} /$ fed. $+100 \mathrm{~kg} \mathrm{~K} 2 \mathrm{O} /$ fed.)

The compost obtained from (Beny Sweef Company to the Organic Fertilizers) was added during the preparation of the experimental soil at the rates of $12,9,6$ and $3 \mathrm{~m}^{3} / \mathrm{fed}$ which represented 100,75 , 50 and $25 \%$ respectively. The chemical composition of the dry compost used, is shown in Table (1).

Effective microorganisms $\left(\mathrm{EM}_{1}\right)$ was applied to the soil at the concentration of 5 liters/fed. for two times, the first time was one month after the date of sowing, while, the second one was after one month from the first addition. The content of effective microorganisms $\left(\mathrm{EM}_{1}\right)$ was shown in Table (2) according to Daly and Stewart, (1999) and Higa, (2002).

Preparation of humic acid from vegetarian humic acid fertilizer which contains $86 \%$ humic acid (Abo-Zaabal Company for Fertilizers Manufacturing). Every $100 \mathrm{~g}$ vegetarian humic acid fertilizer containing $86 \mathrm{~g}$ humic acid. The vegetarian humic acid fertilizer was added to the experimental units (soil adding) as a rate of $280 \mathrm{~g} /$ fed. which contains $241.5 \mathrm{~g}$ humic acid, one month from planting and repeated one month later, while humic acid (foliar spraying with $4 \mathrm{~cm} / \mathrm{L}$.) was sprayed on the areal parts of Roselle plants one month from planting and repeated one month later, too.

The source of the microelements was the commercial product Microcomplex, which contains iron at $7 \%$, zinc at $3.5 \%$, manganese at $2.5 \%$, cupper at $0.5 \%$, boron at $0.2 \%$ and molybdenum at $0.1 \%$ was produced by The El-Nokhba Company for the Manufacture of Agricultural Fertilizers and Veterinary Medicines (Nuvac), Amman, Jordan. The microelement product (Microcomplex) used as foliar spraying and as soil adding with $1 \mathrm{~kg} /$ fed. one month from planting and repeated one month later to both applications.

The Roselle plants were chemically fertilized by ammonium sulphate $(20.5 \mathrm{~N} \%)$, calcium superphosphate $\left(15.5 \mathrm{P}_{2} \mathrm{O}_{5} \%\right)$ and potassium sulphate $\left(48 \mathrm{~K}_{2} \mathrm{O} \%\right)$. The nitrogen and potassium fertilizers were applied as soil dressing beside drippers. The amount of nitrogen and potassium fertilizers were divided into three equal doses, the first dose was added after one month from planting, whereas the other two doses were applied 20 days between them and between the first one. While calcium superphosphate fertilizer was added during soil preparation. 
Table 1: Compost analysis.

\begin{tabular}{llll}
\hline Fresh weight $\left(\mathbf{m}^{\mathbf{3}}\right)$ & $\mathbf{7 5 0}-\mathbf{8 5 0} \mathbf{~ k g}$ & Macro elements (\%) & \\
\hline Dry weight $\left(\mathbf{m}^{\mathbf{3}}\right)$ & $550-650 \mathrm{~kg}$ & $\mathbf{N} \%$ & $0.8-1.2$ \\
Moisture \% & $25-30 \%$ & $\mathbf{P} \%$ & $0.4-0.6$ \\
$\mathbf{p H}$ & $7.5-8.5$ & $\mathbf{K} \%$ & $0.8-1.4$ \\
EC ds/m & $3.5-6.5$ & Micro elements (ppm) & \\
Saturation \% & $150-200$ & $\mathbf{F e}$ & $1000-1800$ \\
Organic matter \% & $25-30$ & $\mathbf{M n}$ & $80-120$ \\
Organic carbon \% & $14.5-17.5$ & $\mathbf{C u}$ & $100-160$ \\
C/N ratio & $1: 14.5$ or $1: 18$ & $\mathbf{Z n}$ & $30-50$ \\
\hline
\end{tabular}

Table 2: The content of Effective Microorganisms $\left(\mathrm{EM}_{1}\right)$.

\begin{tabular}{ll}
\hline Photosynthetic bacteria & $\begin{array}{l}\text { Rhodopseudomonas palustrus } \\
\text { Rhodobacter spaeroides }\end{array}$ \\
\hline \multirow{2}{*}{ Lactic acid bacteria } & $\begin{array}{l}\text { Lactobacillus plantarum } \\
\text { Lactobacillus casei } \\
\text { Streptococcus lactis }\end{array}$ \\
\hline Yeasts & $\begin{array}{l}\text { Saccharomyces cerevisiae } \\
\text { Candida utilis }\end{array}$ \\
Actinomycetes & Streptomyces albus \\
Fungi & Streptomyces griseus \\
& Aspergillus oryzae \\
& Mucor hiemalis \\
\hline
\end{tabular}

\subsection{Recorded data}

\subsubsection{Vegetative growth characters}

After 132 days from sowing (in flowering stage), the plant height $(\mathrm{cm})$ and number of branches/plant were recorded, using three random plants from each replicate.

\subsubsection{Yield components}

In both seasons at harvest, when plants reached suitable maturity (180 days after seed sowing), the following data were recorded: number of fruits/plant, fresh weight of fruits $(\mathrm{g}) / \mathrm{plant}$, seeds weight (g)/plant, dry weight of calyx (g)/plant and yield of dry calyx $(\mathrm{kg}) / \mathrm{fed}$.

\subsubsection{Anthocyanin content}

Total anthocyanin content was extracted from dried air harvested Roselle calyxes by using acidified methanol $(1 \% \mathrm{HCl})$ and determined according to the method described by Fuleki and Francis (1968) and developed by Du and Francis (1973).

\subsection{Chemical Analysis}

Chemical analysis of samples during the first and second seasons of the Roselle plant leaves dried at $70{ }^{\circ} \mathrm{C}$ was carried out at the flowering stage. The following determinations were carried out:

- Total nitrogen percentage was determined according to the methods described by Mazumder and Majumder (2003).

- Total phosphorus percentage was determined according to the method described by Jackson (1967).

- Total potassium percentage determination was done photometrically according to the method described by Mazumder and Majumder (2003).

\subsection{Insects, mite pests and associated natural enemies sampling}

In this study more than one sampling methods were used to survey and estimate population densities of pests and associated natural enemies on Roselle plants. The plant sample method: where weekly samples of ten seedlings or ten leaves of mature plants in each of the three replicates plot were selected randomly from different levels of plants canopy and inspected actually early in the morning in the field using hand lens (10x), to count individuals of all stages of pests in all treatments. The mean numbers of stink green bug and natural enemies (except mite predators which counted on leaves with phytophagous mite) were estimated based on the branches number/plant. The sweeping net (with a 
diameter of $30 \mathrm{~cm}$ and $70 \mathrm{~cm}$ in depth) method was used to provide true indicator about flight insects especially leafhoppers, weekly 5 strokes were took from each plot; the caught insect species were transferred to plastic sacks, tied by rubber bands and taken to the laboratory for identification, counting and recording. The sampling operation was started just after emergence complete and continued till harvest. In case of each of cotton seed bug and spiny boll worms, samples of 10 bolls/replicate were collected in randomized and inspected throughout the flowering and fruiting period.

\subsection{Statistical analysis}

The obtained data were statistically analyzed according to Gomez and Gomez (1984) and mean values of the treatments were compared by Duncan's Multiple Range Test (Duncan, 1958), at 5\% probability level.

\section{Results and Discussion}

\subsection{Vegetative growth}

The data tabulated in Table (3) show that, there were positive effects due to using the organic, bio and chemical fertilizers on plant height and number of branches/plant of Hibiscus sabdariffa plant. The tallest plant and the highest number of branches/plant obtained from the plant treated with the treatment of $(25 \%$ Compost $+75 \%$ NPK) followed by (Compost + EM land + Humic acid land + Microelements land) and (100\% NPK) by non-significant differences between them, during the two seasons. While, there were significant differences between these treatments and control $(100 \%$ compost), during the two seasons.

Table 3: Effect of chemical, bio and organic fertilizers on vegetative growth characters.

\begin{tabular}{|c|c|c|c|c|c|c|c|c|}
\hline \multirow{3}{*}{$\begin{array}{l}\text { Treatments } \\
\text { Compost* }\end{array}$} & \multicolumn{4}{|c|}{ Plant height } & \multicolumn{4}{|c|}{ No. of branches/plant } \\
\hline & \multicolumn{2}{|c|}{$1^{\text {st }}$ season } & \multicolumn{2}{|c|}{$2^{\text {nd }}$ season } & \multicolumn{2}{|c|}{$1^{\text {st }}$ season } & \multicolumn{2}{|c|}{$2^{\text {nd }}$ season } \\
\hline & 190.7 & $\mathrm{~d}$ & 200.9 & $\mathrm{f}$ & 6.7 & $\mathrm{c}$ & 5.0 & $\mathrm{c}$ \\
\hline Compost* + EM land & 201.7 & $\mathrm{~cd}$ & 202.3 & ef & 6.7 & $\mathrm{c}$ & 7.3 & $\mathrm{bc}$ \\
\hline Compost* + Humic land & 214.6 & bc & 208.0 & def & 8.7 & $\mathrm{bc}$ & 8.0 & bc \\
\hline Compost* + Humic foliar & 214.7 & bc & 211.0 & cdef & 9.3 & $a b c$ & 8.0 & bc \\
\hline Compost* + elements land & 214.3 & bc & 207.7 & def & 8.3 & $\mathrm{bc}$ & 7.7 & bc \\
\hline Compost* + elements foliar & 217.3 & bc & 215.7 & bcdef & 9.3 & $\mathrm{abc}$ & 8.3 & $\mathrm{ab}$ \\
\hline Compost* + EM land + Humic land & 221.7 & $a b c$ & 219.7 & bcde & 10.0 & $a b$ & 9.7 & $\mathrm{ab}$ \\
\hline Compost* + EM land + elements land & 222.7 & $a b c$ & 220.3 & bcd & 10.3 & $\mathrm{ab}$ & 9.7 & $\mathrm{ab}$ \\
\hline Compost* + Humic foliar + elements foliar & 218.3 & $\mathrm{bc}$ & 218.3 & bcdef & 9.7 & $a b$ & 9.0 & $\mathrm{ab}$ \\
\hline Compost* + Humic Land + elements land & 220.3 & $a b c$ & 219.3 & bcde & 10.0 & $a b$ & 9.0 & $\mathrm{ab}$ \\
\hline $\begin{array}{l}\text { Compost* }+ \text { EM land }+ \text { Humic Land }+ \\
\text { elements land }\end{array}$ & 227.5 & $a b$ & 229.3 & $\mathrm{~b}$ & 12.0 & $\mathrm{a}$ & 10.3 & $\mathrm{ab}$ \\
\hline $75 \%$ Compost $+25 \%$ NPK & 221.0 & $a b c$ & 225.2 & bcd & 10.3 & $\mathrm{ab}$ & 9.7 & $\mathrm{ab}$ \\
\hline $50 \%$ Compost $+50 \%$ NPK & 223.0 & $\mathrm{abc}$ & 226.0 & bc & 10.3 & $a b$ & 10.0 & $\mathrm{ab}$ \\
\hline $25 \%$ Compost $+75 \%$ NPK & 240.0 & $\mathrm{a}$ & 244.8 & $\mathrm{a}$ & 12.0 & $\mathrm{a}$ & 11.7 & $\mathrm{a}$ \\
\hline Zero Compost $+100 \%$ NPK & 223.3 & $a b c$ & 226.0 & $\mathrm{bc}$ & 10.7 & $\mathrm{ab}$ & 10.3 & $\mathrm{ab}$ \\
\hline
\end{tabular}

A similar trend was resulted by Abou El-Hassan et al (2017) in Egypt, stated that, compost + $50 \%$ mineral fertilizers (NPK) were not showed any significant differences compared to $100 \%$ mineral fertilizers on snap bean (Phaseolus vulgaris L.). Also, in Egypt, Hewidy et al (2018) mentioned that, the application of compost on Roselle plants had a positive effect on plant height and number of branches.

\subsection{Fruits number/plant}

As for described in the Table (4) we can reveal that, the treatment of $(25 \%$ Compost $+75 \%$ NPK) gave the highest number of fruits/plant, over the treatments of (Compost + EM land + Humic acid Land + Microelements land) and (100\% compost) with significant differences between them, in the first season. While, the treatment of (Compost + EM land + Humic acid Land + Microelements 
land) raised over $(100 \%$ compost $)$ treatment with significant difference between them in the two seasons.

Beside the stimulated effects of compost, EM and Humic acid, similar findings were obtained by Khattab (1997) applied the foliar nutrient Fetrilon-Combi green (FCg) to Roselle (Hibiscus sabdariffa) as 2, 3 or 4 sprays and found that, fruit yield as well as sepals and seed yield significantly increased with increasing FCg application.

Table 4: Effect of chemical, bio and organic fertilizers on fruit characters and seed yield.

\begin{tabular}{|c|c|c|c|c|c|c|c|c|c|c|c|c|}
\hline \multirow{2}{*}{ Treatments } & \multicolumn{4}{|c|}{ Fruits number/plant } & \multicolumn{4}{|c|}{$\begin{array}{l}\text { Fresh weight of fruits } \\
\text { (g)/plant }\end{array}$} & \multicolumn{4}{|c|}{ Seed yield $(g) /$ plant } \\
\hline & \multicolumn{2}{|c|}{$1^{\text {st }}$ season } & \multicolumn{2}{|c|}{$2^{\text {nd }}$ season } & \multicolumn{2}{|c|}{$1^{\text {st }}$ season } & \multicolumn{2}{|c|}{$2^{\text {nd }}$ season } & \multicolumn{2}{|c|}{$1^{\text {st }}$ season } & \multicolumn{2}{|c|}{$\begin{array}{c}2^{\text {nd }} \\
\text { season }\end{array}$} \\
\hline Compost* & 6.7 & $\mathrm{~g}$ & 11.7 & $\mathrm{e}$ & 97.4 & $\mathrm{f}$ & 116.7 & ef & 9.7 & $\mathrm{f}$ & 11.8 & $\mathrm{c}$ \\
\hline Compost* + EM land & 10.0 & $\mathrm{~g}$ & 20.3 & d & 104.7 & ef & 130.7 & def & 10.5 & ef & 12.1 & $\mathrm{c}$ \\
\hline Compost* + Humic land & 20.3 & ef & 22.3 & $\mathrm{~cd}$ & 111.7 & def & 131.5 & def & 13.0 & def & 13.4 & $\mathrm{c}$ \\
\hline Compost* + Humic foliar & 21.0 & ef & 22.7 & $\mathrm{~cd}$ & 112.0 & def & 138.0 & def & 14.0 & def & 14.2 & $\mathrm{c}$ \\
\hline Compost* + elements land & 20.0 & $\mathrm{f}$ & 22.3 & $\mathrm{~cd}$ & 104.7 & ef & 131.5 & def & 11.0 & ef & 13.5 & $\mathrm{c}$ \\
\hline Compost* + elements foliar & 22.7 & def & 24.5 & $\mathrm{~cd}$ & 119.6 & cdef & 143.0 & cdef & 14.4 & cdef & 15.6 & $\mathrm{bc}$ \\
\hline $\begin{array}{l}\text { Compost } *+\text { EM land + Humic } \\
\text { land }\end{array}$ & 26.5 & cde & 26.5 & $\mathrm{~cd}$ & 154.0 & bcde & 160.7 & cdef & 16.4 & bcd & 17.4 & $\mathrm{bc}$ \\
\hline $\begin{array}{l}\text { Compost } *+\text { EM land }+ \\
\text { elements land }\end{array}$ & 27.3 & $\mathrm{~cd}$ & 28.0 & bcd & 163.3 & bcd & 172.7 & cdef & 16.8 & bcd & 17.5 & bc \\
\hline $\begin{array}{l}\text { Compost } *+\text { Humic foliar }+ \\
\text { elements foliar }\end{array}$ & 25.7 & cdef & 25.7 & $\mathrm{~cd}$ & 120.0 & cdef & 146.7 & cdef & 14.6 & cdef & 15.9 & bc \\
\hline $\begin{array}{l}\text { Compost* + Humic Land + } \\
\text { elements land }\end{array}$ & 25.9 & cdef & 25.7 & $\mathrm{~cd}$ & 120.7 & cdef & 151.3 & cdef & 15.3 & cde & 16.6 & bc \\
\hline $\begin{array}{l}\text { Compost* + EM land + Humic } \\
\text { Land + elements land }\end{array}$ & 40.3 & $\mathrm{~b}$ & 43.0 & a & 197.0 & $\mathrm{~b}$ & 257.7 & $a b$ & 37.2 & $\mathrm{a}$ & 30.9 & $\mathrm{a}$ \\
\hline 75\% Compost + 25\% NPK & 27.3 & $\mathrm{~cd}$ & 29.3 & $\mathrm{bc}$ & 167.3 & $\mathrm{bc}$ & 192.9 & bcde & 19.1 & $\mathrm{bc}$ & 17.6 & $\mathrm{bc}$ \\
\hline $50 \%$ Compost $+50 \%$ NPK & 27.7 & cd & 29.5 & bc & 177.3 & $\mathrm{~b}$ & 218.0 & abcd & 20.9 & $\mathrm{~b}$ & 18.5 & $\mathrm{bc}$ \\
\hline $25 \%$ Compost $+75 \%$ NPK & 47.7 & a & 48.3 & $\mathrm{a}$ & 287.7 & a & 282.7 & a & 38.3 & $\mathrm{a}$ & 35.6 & $\mathrm{a}$ \\
\hline Zero Compost + $100 \%$ NPK & 31.3 & $\mathrm{c}$ & 35.0 & $\mathrm{~b}$ & 179.3 & $\mathrm{~b}$ & 227.3 & $a b c$ & 21.1 & $\mathrm{~b}$ & 27.7 & $\mathrm{ab}$ \\
\hline
\end{tabular}

\subsection{Fresh weight of fruits $(\mathrm{g}) / \mathrm{plant}$}

The effects of organic, bio and chemical fertilizers on fresh weight of fruits (g)/plant were presented in Table (4) and showed that, the treatments of (Compost + EM land + Humic acid land + Microelements land) raised the fresh weight over the treatment of $(100 \% \mathrm{NPK})$ with no significant difference between them. In the same trend, the treatment of (Compost + EM land + Humic acid land + Microelements land) gave significant differences with other treatments, during the $1^{\text {st }}$ and $2^{\text {nd }}$ seasons, except the treatments of (Compost + EM land + Humic land) and (Compost + EM land + elements land) in the first season. On the other hand, the treatment of $(25 \%$ Compost $+75 \%$ NPK $)$ gave the highest and significant fruits number comparing with all treatments, in the two seasons with no significant difference between the treatment of (Compost + EM land + Humic acid land + Microelements land) and $(100 \%$ NPK $)$ in the second season.

Similar results, in general, were obtained by Hu and Qi (2013) stated that, the application of $\mathrm{EM}_{1}$ in combination with compost significantly increased wheat grain yields.

These results may be due to the compost have multiple positive effects on the physical, chemical and biological soil properties, so, it contributes to the stabilization and increase of crop productivity and crop quality (Amlinger et al., 2007 and Tayebeh et al., 2010). And, Humic acid promote chemical reaction for cation exchange, increase $\mathrm{pH}$ buffering capacity of soils, bind and sequester phytotoxic elements and accelerate transport of nutrients to plants (Amjad et al., 2010).

\subsection{Seed yield (g)/plant}

Concerning the seed yield (g)/plant as affected by different combination between organic, bio and chemical fertilizers treatments, the data presented in Table (4) stated that, the treatment of $(25 \%$ Compost $+75 \%$ NPK) gave the heaviest seed yield compared with the other treatments with significant differences, except with the treatment of (Compost + EM land + Humic acid land + Microelements 
land) with non-significant difference between them. Both these treatments significantly raised the seed yield/plant over the treatment of $(100 \% \mathrm{NPK})$ and other treatments, in the first season.

Similarly, when El-Sherif (2016) used $400 \mathrm{~g} /$ fed. foliar micronutrients on sesame (Sesamum indicum $\mathrm{L}$.) led to increase number of capsules plant ${ }^{-1}$, weight of capsules plant ${ }^{-1}(\mathrm{~g})$ and seed yield plant ${ }^{1}(\mathrm{~g})$.

\subsection{Dry sepals yield}

Results in Table (5) indicate that, the different organic, bio and chemical fertilizers treatment affected the yield of dry sepals in Roselle plant. The highest dry sepals weight (g)/plant and yield of dry sepals $(\mathrm{kg}) / \mathrm{fed}$. observed when using the treatment of $(25 \%$ Compost $+75 \%$ NPK $)$ followed by (Compost + EM land + Humic acid land + Microelements land) and (100\% NPK), respectively, with no significant differences between them, but there were significant differences with other treatments, in both seasons. The treatment of (Compost + EM land + Humic acid land + Microelements land) raised the weight of dry sepals (g)/plant over using the (Compost only) treatment, with 144.7 and $106.2 \%$, and resulted 200.0 and $222.8 \mathrm{~kg} /$ fed of dry sepals, while the treatment of $(25 \%$ Compost $+75 \% \mathrm{NPK})$ yielded 234.0 and $270.0 \mathrm{~kg} / \mathrm{fed}$, with 17.00 and $21.2 \%$ rising weight of dry sepals $(\mathrm{g}) / \mathrm{plant}$ over the previous treatment, during the first and second seasons, respectively.

Similar results were found by Khalil and Yousef (2014) revealed that, dual application of both organic and inorganic fertilizers caused significant increases in the growth, yield and fruit quality of Roselle plants compared with the individual treatment. Also, Hewidy et al (2018) mentioned that, adding compost on Roselle plants had a positive effect on number of fruits and dry weight of calyx. In the same manner, Hanafy (2009) stated that, yield of dry sepals/plant were obtained with foliar spray by micro-elements at $90 \mathrm{ppm}$. on Roselle plant.

Table 5: Effect of chemical, bio and organic fertilizers on dry sepals yield and anthocyanin content.

\begin{tabular}{|c|c|c|c|c|c|c|c|c|c|c|c|c|}
\hline \multirow{3}{*}{$\begin{array}{l}\text { Treatments } \\
\text { Compost* }\end{array}$} & \multicolumn{4}{|c|}{$\begin{array}{c}\text { Dry weight of sepals } \\
\text { (g)/plant }\end{array}$} & \multicolumn{4}{|c|}{$\begin{array}{c}\begin{array}{c}\text { Yield of dry sepals } \\
(\mathrm{kg}) / \mathrm{fed}\end{array} \\
\end{array}$} & \multicolumn{4}{|c|}{$\begin{array}{c}\text { Anthocyanin content } \\
(\mathrm{mg} / 100 \mathrm{ml})\end{array}$} \\
\hline & \multicolumn{2}{|c|}{$1^{\text {st }}$ season } & \multicolumn{2}{|c|}{$2^{\text {nd }}$ season } & \multicolumn{2}{|c|}{$1^{\text {st }}$ season } & \multicolumn{2}{|c|}{$2^{\text {nd }}$ season } & \multicolumn{2}{|c|}{$1^{\text {st }}$ season } & \multicolumn{2}{|c|}{$2^{\text {nd }}$ season } \\
\hline & 7.6 & $\mathrm{~d}$ & 8.1 & $\mathrm{c}$ & 90.7 & $\mathrm{~d}$ & 97.6 & $\mathrm{c}$ & 10.24 & $\mathrm{~h}$ & 10.12 & g \\
\hline Compost* + EM land & 7.7 & $\mathrm{~d}$ & 8.5 & $\mathrm{c}$ & 92.0 & $\mathrm{~d}$ & 101.6 & c & 11.80 & gh & 13.27 & $\mathrm{fg}$ \\
\hline Compost ${ }^{*}+$ Humic land & 9.4 & $\mathrm{~cd}$ & 8.9 & $\mathrm{bc}$ & 113.2 & $\mathrm{~cd}$ & 107.2 & $\mathrm{bc}$ & 14.79 & efg & 14.62 & ef \\
\hline Compost $*+$ Humic foliar & 9.5 & $\mathrm{~cd}$ & 8.9 & $\mathrm{bc}$ & 113.6 & $\mathrm{~cd}$ & 107.2 & $\mathrm{bc}$ & 16.71 & def & 14.92 & ef \\
\hline Compost $*$ + elements land & 9.2 & $\mathrm{~cd}$ & 8.7 & $\mathrm{bc}$ & 110.4 & $\mathrm{~cd}$ & 104.8 & $\mathrm{bc}$ & 14.54 & gh & 13.78 & $\mathrm{f}$ \\
\hline Compost* + elements foliar & 9.6 & $\mathrm{~cd}$ & 9.4 & $\mathrm{bc}$ & 115.2 & $\mathrm{~cd}$ & 112.8 & $\mathrm{bc}$ & 16.71 & def & 15.73 & def \\
\hline $\begin{array}{l}\text { Compost }^{*}+\text { EM land }+ \\
\text { Humic land }\end{array}$ & 12.1 & bcd & 10.3 & $\mathrm{bc}$ & 145.2 & bcd & 124.0 & bc & 18.18 & cde & 19.72 & bc \\
\hline $\begin{array}{l}\text { Compost* }+ \text { EM land }+ \\
\text { elements land }\end{array}$ & 12.3 & bcd & 11.5 & $\mathrm{bc}$ & 147.2 & bed & 138.0 & $\mathrm{bc}$ & 18.27 & cde & 19.76 & $\mathrm{bc}$ \\
\hline $\begin{array}{l}\text { Compost* + Humic foliar }^{*}+\text { elements foliar }\end{array}$ & 10.5 & $\mathrm{~cd}$ & 9.7 & $\mathrm{bc}$ & 126.4 & $\mathrm{~cd}$ & 116.0 & $\mathrm{bc}$ & 17.40 & cdef & 18.00 & cde \\
\hline $\begin{array}{l}\text { Compost } *+\text { Humic Land + } \\
\text { elements land }\end{array}$ & 11.6 & $\mathrm{~cd}$ & 9.9 & $\mathrm{bc}$ & 139.2 & $\mathrm{~cd}$ & 118.8 & $\mathrm{bc}$ & 17.93 & cdef & 18.60 & $\mathrm{~cd}$ \\
\hline $\begin{array}{l}\text { Compost* }+ \text { EM land }+ \\
\text { Humic Land }+ \text { elements } \\
\text { land }\end{array}$ & 18.6 & a & 16.7 & $a b$ & 200.0 & $\mathrm{a}$ & 222.8 & $a b$ & 23.10 & $\mathrm{ab}$ & 24.08 & $\mathrm{a}$ \\
\hline $75 \%$ Compost $+25 \%$ NPK & 12.7 & $\mathrm{bc}$ & 11.7 & $\mathrm{bc}$ & 152.8 & $\mathrm{bc}$ & 140.0 & $\mathrm{bc}$ & 19.98 & bed & 19.93 & $\mathrm{bc}$ \\
\hline $50 \%$ Compost $+50 \%$ NPK & 12.8 & $\mathrm{bc}$ & 15.1 & $\mathrm{bc}$ & 154.0 & $\mathrm{bc}$ & 180.8 & $\mathrm{bc}$ & 20.70 & $\mathrm{abc}$ & 20.08 & $\mathrm{bc}$ \\
\hline $25 \%$ Compost $+75 \%$ NPK & 19.5 & $\mathrm{a}$ & 22.5 & a & 234.0 & a & 270.0 & $\mathrm{a}$ & 23.86 & $\mathrm{a}$ & 25.23 & $\mathrm{a}$ \\
\hline $\begin{array}{ll}\text { Zero } & \text { Compost }+100 \% \\
\text { NPK } & \\
\end{array}$ & 16.5 & $a b$ & 15.7 & $\mathrm{abc}$ & 198.4 & $a b$ & 188.0 & $a b c$ & 20.96 & $a b c$ & 22.73 & $a b$ \\
\hline
\end{tabular}

\subsection{Anthocyanin content $(\mathrm{mg} / \mathbf{1 0 0} \mathrm{ml})$}

Anthocyanin content of Roselle calyces as affected by different organic, bio and chemical fertilizers treatments are shown in Table (5). Data demonstrate that, the highest content of anthocyanin resulted from the treatment of ( $25 \%$ Compost $+75 \%$ NPK) followed by the treatments of (Compost + EM land + Humic acid land + Microelements land) and (100\% NPK), in the $1^{\text {st }}$ and $2^{\text {nd }}$ seasons, respectively, with no significant differences between them, during the two seasons. 


\subsection{NPK percentages}

Data presented in Table (6) record the effect of different combination between organic, bio and chemical fertilizers treatments, on NPK percentages of Roselle leaves. The data show that, the highest nitrogen, phosphorus and potassium percentages resulted from the treatment of ( $25 \%$ Compost $+75 \%$ NPK), followed by $(100 \% \mathrm{NPK})$ and $(50 \%$ Compost $+50 \% \mathrm{NPK})$ treatments, respectively, during the two seasons.

Table 6: Effect of chemical, bio and organic fertilizers on NPK percentages.

\begin{tabular}{|c|c|c|c|c|c|c|c|c|c|c|c|c|}
\hline \multirow{3}{*}{$\begin{array}{l}\text { Treatments } \\
\text { Compost* }\end{array}$} & \multicolumn{4}{|c|}{ Nitrogen \% } & \multicolumn{4}{|c|}{ Phosphorus \% } & \multicolumn{4}{|c|}{ Potassium \% } \\
\hline & \multicolumn{2}{|c|}{$\begin{array}{c}1^{\text {st }} \\
\text { season }\end{array}$} & \multicolumn{2}{|c|}{$\begin{array}{c}2^{\text {nd }} \\
\text { season }\end{array}$} & \multicolumn{2}{|c|}{$1^{\text {st }}$ season } & \multicolumn{2}{|c|}{$2^{\text {nd }}$ season } & \multicolumn{2}{|c|}{$\begin{array}{c}1^{\text {st }} \\
\text { season }\end{array}$} & \multicolumn{2}{|c|}{$\begin{array}{c}2^{\text {nd }} \\
\text { season }\end{array}$} \\
\hline & 1.54 & $\mathrm{~m}$ & 1.57 & $\mathrm{n}$ & 0.367 & $\mathrm{k}$ & 0.393 & $\mathrm{j}$ & 1.64 & o & 1.67 & o \\
\hline Compost* + EM land & 1.61 & 1 & 1.65 & $\mathrm{~m}$ & 0.410 & $\mathrm{j}$ & 0.433 & $\mathrm{i}$ & 1.69 & $\mathrm{n}$ & 1.73 & $\mathrm{n}$ \\
\hline Compost $*+$ Humic land & 1.72 & $\mathrm{k}$ & 1.74 & 1 & 0.433 & $\mathrm{i}$ & 0.447 & hi & 1.76 & $\mathrm{~m}$ & 1.79 & $\mathrm{~m}$ \\
\hline Compost* + Humic foliar & 1.91 & $\mathrm{i}$ & 1.96 & $\mathrm{j}$ & 0.453 & $\mathrm{~h}$ & 0.463 & $\mathrm{~g}$ & 1.86 & $\mathrm{k}$ & 1.91 & $\mathrm{k}$ \\
\hline Compost* + Elements land & 1.84 & $\mathrm{j}$ & 1.90 & $\mathrm{k}$ & 0.463 & gh & 0.457 & gh & 1.83 & 1 & 1.88 & 1 \\
\hline Compost* + Elements foliar & 1.98 & $\mathrm{~h}$ & 2.04 & $\mathrm{i}$ & 0.467 & fg & 0.483 & $\mathrm{f}$ & 2.08 & $\mathrm{j}$ & 2.13 & $\mathrm{j}$ \\
\hline Compost* + EM land + Humic land & 2.10 & $\mathrm{~g}$ & 2.15 & $\mathrm{~h}$ & 0.477 & $\mathrm{f}$ & 0.487 & $\mathrm{f}$ & 2.21 & $\mathrm{i}$ & 2.29 & $\mathrm{i}$ \\
\hline Compost* + EM land + Elements land & 2.14 & $\mathrm{f}$ & 2.18 & g & 0.507 & $\mathrm{e}$ & 0.503 & de & 2.32 & $\mathrm{~h}$ & 2.40 & $\mathrm{~h}$ \\
\hline $\begin{array}{l}\text { Compost* }+ \text { Humic foliar }+ \text { Elements } \\
\text { foliar }\end{array}$ & 2.21 & $\mathrm{e}$ & 2.24 & $\mathrm{f}$ & 0.513 & e & 0.497 & ef & 2.48 & $\mathrm{~g}$ & 2.57 & g \\
\hline $\begin{array}{l}\text { Compost* + Humic Land + Elements } \\
\text { land }\end{array}$ & 2.26 & $\mathrm{~d}$ & 2.29 & e & 0.517 & de & 0.517 & d & 2.59 & $\mathrm{f}$ & 2.68 & $\mathrm{f}$ \\
\hline $\begin{array}{l}\text { Compost* + EM land + Humic Land + } \\
\text { Elements land }\end{array}$ & 2.31 & c & 2.35 & d & 0.527 & $\mathrm{~cd}$ & 0.543 & $\mathrm{c}$ & 2.64 & $\mathrm{e}$ & 2.70 & $\mathrm{e}$ \\
\hline $75 \%$ Compost + 25\% NPK & 2.32 & c & 2.41 & c & 0.537 & $\mathrm{c}$ & 0.553 & $\mathrm{bc}$ & 2.72 & d & 2.74 & $\mathrm{~d}$ \\
\hline $50 \%$ Compost $+50 \%$ NPK & 2.35 & $\mathrm{~b}$ & 2.45 & $\mathrm{~b}$ & 0.537 & $\mathrm{c}$ & 0.557 & $\mathrm{bc}$ & 2.79 & c & 2.81 & c \\
\hline $25 \%$ Compost + 75\% NPK & 2.42 & $\mathrm{a}$ & 2.53 & a & 0.573 & a & 0.613 & $\mathrm{a}$ & 2.91 & a & 2.95 & $\mathrm{a}$ \\
\hline Zero Compost + $100 \%$ NPK & 2.35 & $\mathrm{~b}$ & 2.47 & $\mathrm{~b}$ & 0.557 & $\mathrm{~b}$ & 0.567 & $\mathrm{~b}$ & 2.83 & $\mathrm{~b}$ & 2.87 & $\mathrm{~b}$ \\
\hline
\end{tabular}

\subsection{Pests and associated natural enemies}

\subsubsection{Survey of pests infested Roselle plan plants}

The results in Table (7) reveal that, the Roselle plants found infested the tested treatments with more than one species of leafhoppers, Empoasca spp.; three species of aphids [cotton aphids, Aphis gossypii (Glov.); green peach aphids, Myzus persicae (Sulz) and cow bean aphids, Aphis craccivora]; cotton whitefly, Bemisia tabaci (Genn.); onion thrips, Thrips tabaci (Lind.); green stink bug, Nezara viridula L; cotton seeds bug, Oxycarenus hyalinipennis (Costa); spiny boll worm, Earias insulana (Boisd.) and the two spotted spider mite, Tetranychus urticea (Koch). The main and dominant piercingsucking pests recorded relatively high numbers were leafhoppers, Empoasca spp (8.3 individuals/leaf), A. gossypii (40.7 individuals/leaf) and T. urticea (32.3 individuals/leaf) found infesting Roselle plants followed by $B$. tabaci (15.3 individuals/leaf) and T. tabaci (10.3 individuals/leaf) during vegetative growth period; O. hyalinipennis (97 individuals/leaf) during fruiting period of Roselle plants.

\subsubsection{Survey of associated natural enemies:}

The obtained data in Table (7) show that, four species of insect predators [lady bird beetle, Coccinella spp.; green lace wing, Chrisoprella carnea (Steph.); Scymnus beetles, Scymnus spp. and orius bug, Orius albidipennis Reut.] and tow predator mites [Phytoseiulus sp. and Euseius sp.] were found associated with pests infested Roselle plants under different chemical, bio and organic fertilizers treatments .

The obtained results of pests and associated natural enemies were found in agreement with those of Mesbah et al. (1982), Ahmed (1990), El-Sayed et al. (1990), Ahmed et al. (1995) and Afsah (2005) who reported that the aphids ( $A$. gossypii), whitefly $(B$. tabaci) were the most abundant piercing-sucking insect during vegetative growth and $O$. hyalinipennis bug during fruiting period on Roselle plants. Also they added that the most common insect predators species were Coccinella spp., Orius albidipennis, Scymnus interruptus goeze and Chriysoprela carnea (steph). In the same trend, the results found in harmony with those of Hammad and Mohsen (2000) recorded C. undcimpunctata, S. interruptus, O. albidipennis, O. laevigatus, P. alfierii and M. corollae as predatory insects on Roselle plants. Also, 
Ismail (2001) reported that, C. carnea, C. undecimpunctata, S. interrptus, $P$. alfierii and $P$. gallica were the most important insect predaceous species on certain medicinal and aromatic plants. Also, with those of Osman et al (2017) who mentioned that, Aphis gossypii Glover, Bemisia tabaci (Genn.), Empoasca decipiens (Paoli), Nezara viridula L. Although, Oxycarinus hyalinipennis (Costa) and Thrips tabaci (Lind.) were the most dominant insect pests on Roselle plants.

Table 7: Survey of piercing-sucking pests and associated natural enemies on Hibiscus sabdariffa L. plants under chemical, bio and organic fertilizers treatments during the two seasons of 2017 and 2018.

\begin{tabular}{|c|c|c|c|c|c|c|}
\hline Groups & English name & Scientific name & Arabic name & Family name & Order name & $\begin{array}{c}\text { Occurrence } \\
\text { level }\end{array}$ \\
\hline \multirow{8}{*}{ 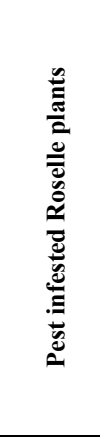 } & $\begin{array}{l}\text { leafhoppers } \\
\text { (jassids) }\end{array}$ & Empoasca spp. & El jassid & Cicadellidae & Homoptera & ++++ \\
\hline & Aphids & $\begin{array}{l}\text { Aphis gossypii (Glov.) } \\
\text { Myzus persicae (sulz) } \\
\text { Aphis craccivora }\end{array}$ & Al men & Aphididae & Homoptera & $\begin{array}{c}+++ \\
+ \\
+ \\
\end{array}$ \\
\hline & Cotton whitefly & Bemisia tabaci (Genn.) & Zobaba bidaa & Alerodidae & Homoptera & ++ \\
\hline & Onion thrips & Thrips tabaci (Lind.) & Treps & Thrpidae & Thaythanoptera & ++ \\
\hline & Green stink bug & Nezara viridula $L$ & $\begin{array}{l}\text { El buka El } \\
\text { hkadraa }\end{array}$ & pentatomidae & Hemeptera & ++ \\
\hline & Cotton seeds bug & $\begin{array}{l}\text { Oxycarenus hyalinipennis } \\
\text { (Costa) }\end{array}$ & $\begin{array}{l}\text { Bukt bezret } \\
\text { elcotton }\end{array}$ & Lygaeidae & Hemeptera & ++ \\
\hline & $\begin{array}{l}\text { Two spotted } \\
\text { spider mite }\end{array}$ & Tetranychus urticea (Koch) & Acaros & Tetranychidae & Acari & ++++ \\
\hline & Spiny boll worm & Earias insulana (Boisd.). & El shawkia & Nolidae & lepidoptera & + \\
\hline \multirow{6}{*}{ 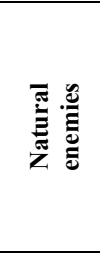 } & Lady birds beetles & Coccinella spp. & Abo El eid & Coccinellidae & Coleoptera & ++ \\
\hline & Green lace wing & Chrisoprella carnea (Steph) & Asad El men & Chrysopidae & Neuroptera & ++ \\
\hline & Scymnus beetles & Scymnus spp. & Scymnus & Coccinellidae & Coleoptera & + \\
\hline & Orius bug & Orius albidipennis reut. & Bukt El oris & anthocoridae & Hemeptera & ++ \\
\hline & \multirow{2}{*}{ Predacious mite } & Phytoseiulus $\mathrm{sp}$. & \multirow{2}{*}{$\begin{array}{l}\text { Moftersate } \\
\text { acaros }\end{array}$} & \multirow{2}{*}{ Phytoseiidae } & \multirow{2}{*}{ Acari } & + \\
\hline & & Euseius sp. & & & & + \\
\hline
\end{tabular}

3.9. Effect of chemical, bio and organic fertilizers on the main pests infested Hibiscus sabdariffa L. plants and its associated natural enemies during the two seasons of 2015 and 2016.

\subsubsection{Effects on the main pests:}

The results presented in Table ( $8 \mathrm{a}$ and $\mathrm{b})$ revealed that, there were significant differences $(\mathrm{P} \leq$ $0.05)$ between the mean numbers of scouted pests on Roselle plants in different fertilizers treatments depending on the variation of chemical, bio and organic fertilizers rates and types, where the lowest mean numbers of the dominant homopterous pests Empoasca spp., Aphis gossypii, Bemisia tabaci and Thrips tabaci were $2.00 \& 2.00$ individual/stroke; $7.67 \& 6.33 ; 1.33 \& 2.00$ and $1.33 \& 1.33$ individual/leaf recorded in (Compost + Humic added as foliar spraying), (Compost + Humic added to soil) or (Compost) treatments during the two study seasons, respectively; In contrary the relatively high numbers of previous pests of $11.00 \& 13.00$ individual/stroke; $40.7 \& 38.7 ; 15.3 \& 13.7$ and $10.3 \& 9.67$ individual/leaf were recorded in $(25 \%$ Compost $+75 \%$ NPK $)$ treatment to Empoasca spp. and (Compost + EM land + Humic land + elements land) treatment to Aphis gossypii, Bemisia tabaci and Thrips tabaci, during the two study seasons, respectively.

In regard to the two heteropterous bugs, $N$. viridula and $O$. hyalinipennis, the lowest mean numbers of $1 \& 1.33$ and $17.00 \& 15.33$ individual/sample were recorded for the two insects in (Compost + EM added to soil) and (Compost) treatments during the two study seasons, respectively. In the other hand, the relatively high mean numbers of the two previous insects of $6.67 \& 5.33$ and $97.00 \& 91.33$ individual/sample were recorded in (Compost + EM land + Humic land + elements land) and (25\% Compost $+75 \%$ NPK) during the two study seasons, respectively, as same as that results of homopterous pests.

The last main and serious pest investigated on Roselle plants was the two spotted spider mite, Tetranychus urticea (Koch) witch recorded relatively lowest mean numbers of 10.00\&8.33 individuals/leaf in (Compost) treatment, while the highest mean numbers of 32.3\&30.7 individuals/leaf were recorded in (Compost + EM land + Humic Land + elements added to soil) during the two study seasons, respectively. 
Generally, the relatively high mean numbers of all inspected pests were associated with high vegetative growth as plant height and branches number which resulted with high quantity and types of nitrogen sources (Compost + Humic foliar + elements foliar), (Compost + Humic Land + elements land), (Compost + EM land + Humic Land + elements land), (75\% Compost $+25 \%$ NPK $),(50 \%$ Compost $+50 \% \mathrm{NPK})$ and $(25 \%$ Compost $+75 \% \mathrm{NPK})$ treatments. In the other hand, the relatively lower numbers of pests were recorded in (Compost), (Compost + EM added to soil) or (Compost + Humic added as foliar spraying) treatments. As previous results it could be noticed that the organic and bio fertilizers can be used as integrated crop management agent in sustainable agriculture systems to get safe food with relatively low cost upon long run.

Table 8a: Effect of chemical, bio and organic fertilizers on insect species infested Roselle plants.

\begin{tabular}{|c|c|c|c|c|c|c|c|c|c|c|c|c|c|c|c|c|}
\hline \multirow{3}{*}{$\begin{array}{l}\text { Treatments } \\
\text { Compost* }^{*}\end{array}$} & \multicolumn{4}{|c|}{ Empoasca spp. } & \multicolumn{4}{|c|}{ A. gossypii } & \multicolumn{4}{|c|}{ B. tabaci } & \multicolumn{4}{|c|}{ T. tabaci } \\
\hline & \multicolumn{2}{|c|}{$1^{\text {st }}$ season } & \multicolumn{2}{|c|}{$2^{\text {nd }}$ season } & \multicolumn{2}{|c|}{$1^{\text {st }}$ season } & \multicolumn{2}{|c|}{$\begin{array}{l}2^{\text {nd }} \\
\text { season }\end{array}$} & \multicolumn{2}{|c|}{$1^{\text {st }}$ season } & \multicolumn{2}{|c|}{$\begin{array}{l}2^{\text {nd }} \\
\text { season }\end{array}$} & \multicolumn{2}{|c|}{$1^{\text {st }}$ season } & \multicolumn{2}{|c|}{$2^{\text {nd }}$ season } \\
\hline & 3.0 & $\mathrm{f}$ & 2.3 & ef & 10.3 & hi & 9.67 & gh & 1.33 & $\mathrm{~h}$ & 2.00 & $\mathrm{~d}$ & 1.33 & $\mathrm{~g}$ & 1.33 & $\mathrm{~h}$ \\
\hline Compost* + EM land & 2.7 & $\mathrm{f}$ & 2.0 & $\mathrm{f}$ & 9.33 & $\mathrm{ij}$ & 8.0 & hi & 2.67 & gh & 3.33 & fg & 1.67 & fg & 2.33 & $\mathrm{~h}$ \\
\hline Compost* + Humic land & 2.0 & $\mathrm{f}$ & 2.7 & ef & 7.67 & $\mathrm{j}$ & 6.33 & $\mathrm{i}$ & 3.00 & gh & 2.33 & $\mathrm{~g}$ & 2.33 & efg & 1.67 & $\mathrm{~h}$ \\
\hline $\begin{array}{l}\text { Compost* }+ \text { Humic } \\
\text { foliar }\end{array}$ & 2.0 & $\mathrm{f}$ & 2.0 & $\mathrm{f}$ & 11.3 & ghi & 10.0 & gh & 4.33 & fg & 3.00 & fg & 2.00 & fg & 2.33 & fgh \\
\hline $\begin{array}{l}\text { Compost* + elements } \\
\text { land }\end{array}$ & 2.0 & $\mathrm{f}$ & 3.3 & ef & 12.7 & gh & 10.7 & $\mathrm{~g}$ & 5.00 & fg & 4.67 & ef & 1.67 & fg & 2.00 & gh \\
\hline $\begin{array}{l}\text { Compost* + elements } \\
\text { foliar }\end{array}$ & 2.3 & $\mathrm{f}$ & 3.7 & ef & 13.7 & $\mathrm{~g}$ & 11.3 & $\mathrm{~g}$ & 5.67 & ef & 3.67 & fg & 2.33 & efg & 1.67 & $\mathrm{~h}$ \\
\hline $\begin{array}{l}\text { Compost* + EM land + } \\
\text { Humic land }\end{array}$ & 2.7 & $\mathrm{f}$ & 3.0 & ef & 18.7 & $\mathrm{f}$ & 16.7 & $\mathrm{f}$ & 6.00 & ef & 4.33 & $\mathrm{f}$ & 3.33 & def & 2.67 & fgh \\
\hline $\begin{array}{l}\text { Compost* + EM land + } \\
\text { elements land }\end{array}$ & 3.0 & $\mathrm{f}$ & 3.7 & ef & 20.0 & $\mathrm{f}$ & 18.7 & $\mathrm{f}$ & 7.33 & ef & 6.33 & ef & 4.67 & $\mathrm{~d}$ & 3.33 & fg \\
\hline $\begin{array}{l}\text { Compost } *+\text { Humic } \\
\text { foliar }+ \text { elements foliar }\end{array}$ & 3.7 & ef & 4.0 & def & 23.7 & $\mathrm{e}$ & 21.7 & $\mathrm{e}$ & 10.7 & $\mathrm{bc}$ & 9.67 & $\mathrm{~cd}$ & 4.00 & def & 3.67 & ef \\
\hline $\begin{array}{l}\text { Compost } *+\text { Humic } \\
\text { Land + elements land }\end{array}$ & 5.3 & de & 4.7 & cde & 27.0 & d & 25.7 & $\mathrm{~d}$ & 12.3 & $\mathrm{bc}$ & 10.7 & $\mathrm{~cd}$ & 6.67 & $\mathrm{c}$ & 5.00 & de \\
\hline $\begin{array}{l}\text { Compost* + EM land + } \\
\text { Humic Land + elements } \\
\text { land }\end{array}$ & 8.3 & $\mathrm{bc}$ & 6.7 & c & 40.7 & $\mathrm{a}$ & 38.7 & $\mathrm{a}$ & 15.3 & $\mathrm{a}$ & 13.7 & $\mathrm{a}$ & 10.3 & $\mathrm{a}$ & 9.67 & $\mathrm{a}$ \\
\hline $\begin{array}{ll}75 \% & \text { Compost }+25 \% \\
\text { NPK } & \end{array}$ & 7.0 & $\mathrm{~cd}$ & 6.3 & $\mathrm{~cd}$ & 30.7 & c & 29.7 & c & 14.0 & $\mathrm{ab}$ & 13.3 & $\mathrm{a}$ & 7.67 & bc & 6.33 & $\mathrm{~cd}$ \\
\hline $\begin{array}{ll}\mathbf{5 0} \% & \text { Compost }+\mathbf{5 0} \% \\
\text { NPK } & \end{array}$ & 9.7 & $a b$ & 11.0 & $\mathrm{~b}$ & 34.3 & $\mathrm{~b}$ & 32.3 & $\mathrm{~b}$ & 12.7 & bc & 11.3 & $\mathrm{bc}$ & 9.33 & $a b$ & 8.33 & $a b$ \\
\hline $\begin{array}{ll}25 \% & \text { Compost + } 75 \% \\
\text { NPK } & \end{array}$ & 11.0 & a & 13.0 & a & 36.3 & $\mathrm{~b}$ & 34.3 & $\mathrm{~b}$ & 13.7 & $a b$ & 12.7 & $\mathrm{ab}$ & 8.67 & $a b$ & 7.33 & $\mathrm{bc}$ \\
\hline $\begin{array}{l}\text { Zero Compost + } 100 \% \\
\text { NPK }\end{array}$ & 5.7 & de & 7.0 & c & 22.7 & e & 21.7 & $\mathrm{e}$ & 10.3 & d & 9.00 & $\mathrm{~cd}$ & 6.67 & $\mathrm{c}$ & 5.67 & d \\
\hline
\end{tabular}

Table 8b: Effect of chemical, bio and organic fertilizers on insect and mite species infested Roselle

\begin{tabular}{|c|c|c|c|c|c|c|c|c|c|c|c|c|}
\hline \multirow{3}{*}{$\begin{array}{l}\text { Treatments } \\
\text { Compost* }\end{array}$} & \multirow{2}{*}{\multicolumn{2}{|c|}{$\begin{array}{l}N . \text { viridula } \\
1^{\text {st }} \text { season }\end{array}$}} & \multirow{2}{*}{\multicolumn{2}{|c|}{$\begin{array}{l}2^{\text {nd }} \\
\text { season }\end{array}$}} & \multicolumn{4}{|c|}{ O. hyalinipennis } & \multirow{2}{*}{\multicolumn{2}{|c|}{$\begin{array}{l}\text { T. urticae } \\
1^{\text {st }} \text { season }\end{array}$}} & \multirow{2}{*}{\multicolumn{2}{|c|}{$\begin{array}{l}2^{\text {nd }} \\
\text { season }\end{array}$}} \\
\hline & & & & & \multicolumn{2}{|c|}{$\begin{array}{l}1^{\text {st }} \\
\text { season }\end{array}$} & \multicolumn{2}{|c|}{$2^{\text {nd }}$ season } & & & & \\
\hline & 1.33 & gh & 2.33 & $\mathrm{~cd}$ & 17 & $\mathrm{~m}$ & 15.33 & 1 & 10.0 & $\mathrm{k}$ & 8.33 & $\mathrm{~h}$ \\
\hline Compost* + EM land & 1.00 & $\mathrm{~h}$ & 1.33 & $\mathrm{~d}$ & 19 & $\mathrm{~m}$ & 17.67 & $\mathrm{k}$ & 11.3 & $\mathrm{k}$ & 9.67 & $\mathrm{~h}$ \\
\hline Compost $^{*}+$ Humic land & 1.33 & gh & 2.67 & $\mathrm{~cd}$ & 23 & 1 & 22.33 & $\mathrm{j}$ & 15.7 & $\mathrm{j}$ & 14.0 & g \\
\hline Compost* + Humic foliar & 1.67 & fgh & 2.33 & $\mathrm{~cd}$ & 27 & $\mathrm{k}$ & 24.00 & $\mathrm{j}$ & 16.3 & ij & 16.3 & f \\
\hline Compost* + elements land & 2.33 & efgh & 3.67 & $\mathrm{bc}$ & 30 & $\mathrm{j}$ & 27.00 & $\mathrm{i}$ & 20.3 & gh & 22.3 & d \\
\hline Compost $*$ + elements foliar & 2.67 & defg & 1.33 & $\mathrm{~d}$ & 30 & $\mathrm{j}$ & 27.33 & $\mathrm{i}$ & 24.7 & ef & 25.3 & $\mathrm{bc}$ \\
\hline Compost* + EM land + Humic land & 2.00 & $\mathrm{fgh}$ & 2.67 & $\mathrm{~cd}$ & 33 & $\mathrm{i}$ & 32.67 & $\mathrm{~h}$ & 27.7 & bed & 24.7 & $\mathrm{c}$ \\
\hline Compost $*$ EM land + elements land & 3.67 & cde & 2.33 & $\mathrm{~cd}$ & 37 & $\mathrm{~h}$ & 34.33 & $\mathrm{~h}$ & 23.0 & $\mathrm{f}$ & 21.7 & d \\
\hline Compost $*$ Humic foliar + elements foliar & 4.33 & bcd & 5.00 & $a b$ & 40 & $\mathrm{~g}$ & 38.33 & $\mathrm{~g}$ & 22.3 & $\mathrm{fg}$ & 20.7 & de \\
\hline Compost" + Humic Land + elements land & 5.67 & $a b$ & 6.00 & $\mathrm{a}$ & 56 & $\mathrm{f}$ & 53.33 & $\mathrm{f}$ & 25.7 & de & 27.3 & $\mathrm{~b}$ \\
\hline Compost $^{*}+$ EM land + Humic Land + el & & & & & & & & & & & & \\
\hline land & 6.67 & $a b$ & 5.33 & $a b$ & 87 & $\mathrm{~b}$ & 80.67 & $\mathrm{~b}$ & 32.3 & a & 30.7 & a \\
\hline $75 \%$ Compost $+25 \%$ NPK & 4.67 & bcd & 6.33 & $a b$ & 77 & $\mathrm{~d}$ & 73.00 & d & 28.7 & $\mathrm{bc}$ & 25.3 & $\mathrm{bc}$ \\
\hline $50 \%$ Compost $+\mathbf{5 0 \%}$ NPK & 3.67 & cde & 2.67 & $\mathrm{~cd}$ & 81 & $\mathrm{c}$ & 77.67 & $\mathrm{c}$ & 26.3 & cde & 25.7 & $\mathrm{bc}$ \\
\hline $25 \%$ Compost $+75 \%$ NPK & 5.33 & $a b c$ & 4.00 & $\mathrm{bc}$ & 97 & $\mathrm{a}$ & 91.33 & $\mathrm{a}$ & 29.7 & $\mathrm{~b}$ & 27.3 & $\mathrm{~b}$ \\
\hline Zero Compost $+\mathbf{1 0 0 \%}$ NPK & 3.00 & def & 3.67 & $\mathrm{bc}$ & 60 & $\mathrm{e}$ & 58.33 & $\mathrm{e}$ & 18.7 & hi & 19.0 & $\mathrm{e}$ \\
\hline
\end{tabular}

$\left(\right.$ Compost $*=100 \%=12 \mathrm{~m}^{3} /$ fed. $)-\left(75 \%\right.$ Compost $=9 \mathrm{~m}^{3} /$ fed. $)-\left(50 \%\right.$ Compost $=6 \mathrm{~m}^{3} /$ fed. $)-(25 \%$ Compost $=3$ $\mathrm{m}^{3} /$ fed.) 
The obtained results agreed with those of Hashem et al. (2017) who decided that the insect pests are untied to challenge the plant, the integration between Chicken manure $\& \mathrm{EM}_{1}, \mathrm{EM}$. Bokashi \& $\mathrm{EM}_{1}$ or EM bokashi with Tracer or ALKANZ will be the most effective ecofriendly strategy to overcome these dramatic conditions to conserve the environment and produce an appreciated yield. Also, with that of Shafique et al., (2016) who stated that, the using of organic additives, antagonistic microorganisms and phytochemicals in controlling pests and soil borne diseases offers an alternate strategy to the prevalent use of synthetic pesticides.

\subsubsection{Effects on the natural enemies}

The main and dominant natural enemies tabulated in Table (9) found associated with pests on Roselle plants were the three insect predators, Coccinella spp., Chrisoprella carnea and Orius albidipennis witch recorded relatively lowest mean numbers of $1.00 \& 2.7 ; 0.33 \& 1.00$ and $2.00 \& 4.00$ individuals/plant in (Compost + Microelements added as foliar spraying), (Compost) or (Compost + Humic added to soil) treatments in association with low preys numbers of pests individuals, while the relatively high mean numbers of $5.7 \& 4.3 ; 4.33 \& 5.00$ and $8.67 \& 6.00$ individuals/plant associated with high numbers of pests infested Roselle plants recorded in (Compost + EM land + Humic Land + elements land) during the two study seasons, respectively.

Generally, the fertilizers treatments were affected the activity as indirect effect by affecting pests numbers and direct effect by providing good shelter for predators.

Table 9: Effect of chemical, bio and organic fertilizers on insect predators associated with Roselle plants.

\begin{tabular}{|c|c|c|c|c|c|c|c|c|c|c|c|c|}
\hline \multirow{3}{*}{$\begin{array}{l}\text { Treatments } \\
\text { Compost* }\end{array}$} & \multicolumn{4}{|c|}{ Coccinella spp. } & \multicolumn{4}{|c|}{ Chrisoprella carnea } & \multicolumn{4}{|c|}{ Orius albidipennis } \\
\hline & \multicolumn{2}{|c|}{$1^{\text {st }}$ season } & \multicolumn{2}{|c|}{$2^{\text {nd }}$ season } & \multicolumn{2}{|c|}{$1^{\text {st }}$ season } & \multicolumn{2}{|c|}{$2^{\text {nd }}$ season } & \multicolumn{2}{|c|}{$1^{\text {st }}$ season } & \multicolumn{2}{|c|}{$2^{\text {nd }}$ season } \\
\hline & 2.3 & bed & 3.0 & abcd & 0.33 & $\mathrm{e}$ & 1.00 & fg & 3.33 & $\mathrm{~g}$ & 5.00 & bc \\
\hline Compost* + EM land & 1.7 & $\mathrm{~cd}$ & 2.3 & bed & 1.33 & cde & 1.67 & def & 2.00 & i & 4.00 & de \\
\hline Compost* + Humic land & 2.0 & $\mathrm{~cd}$ & 3.0 & abcd & 0.78 & e & 2.00 & de & 3.33 & $\mathrm{~g}$ & 5.33 & $a b$ \\
\hline Compost* + Humic foliar & 3.3 & $\mathrm{bc}$ & 1.7 & $\mathrm{~d}$ & 2.00 & bed & 1.00 & fg & 4.33 & ef & 5.00 & $\mathrm{bc}$ \\
\hline Compost* + elements land & 2.7 & bcd & 2.0 & $\mathrm{~cd}$ & 1.00 & de & 0.78 & $\mathrm{~g}$ & 4.00 & efg & 5.33 & $a b$ \\
\hline Compost* + elements foliar & 1.0 & d & 2.7 & abcd & 2.00 & bed & 2.00 & de & 3.67 & fgh & 2.33 & $\mathrm{f}$ \\
\hline Compost* + EM land + Humic & & & & & & & & & & & & \\
\hline $\begin{array}{l}\text { land } \\
\text { Compost* + EM land + elements }\end{array}$ & 2.3 & $\mathrm{~cd}$ & 3.7 & $a b c$ & 0.78 & $\mathrm{e}$ & 1.67 & def & 4.67 & de & 4.67 & bcd \\
\hline $\begin{array}{l}\text { land } \\
\text { Compost* }+ \text { Humic foliar }+\end{array}$ & 2.7 & bed & 4.0 & $a b$ & 2.33 & $\mathrm{bc}$ & 2.00 & de & 5.33 & de & 6.00 & $a b$ \\
\hline elements foliar & 3.0 & bc & 3.3 & abcd & 2.33 & bc & 3.67 & $\mathrm{~b}$ & 4.00 & efg & 2.67 & $\mathrm{f}$ \\
\hline $\begin{array}{l}\text { Compost* }+ \text { Humic Land }+ \\
\text { elements land } \\
\text { Compost } * \text { EM land }+ \text { Humic }\end{array}$ & 2.0 & $\mathrm{~cd}$ & 2.3 & bcd & 2.67 & $\mathrm{~b}$ & 1.33 & efg & 3.67 & fgh & 4.67 & bcd \\
\hline Land + elements land & 5.7 & a & 4.3 & a & 4.33 & $\mathrm{a}$ & 5.00 & a & 8.67 & $\mathrm{a}$ & 6.00 & $a b$ \\
\hline $75 \%$ Compost $+25 \%$ NPK & 4.3 & $a b$ & 2.0 & cd & 2.00 & bed & 1.33 & efg & 3.00 & $\mathrm{~h}$ & 5.00 & $\mathrm{bc}$ \\
\hline $50 \%$ Compost $+\mathbf{5 0} \%$ NPK & 2.7 & bed & 3.0 & abcd & 3.00 & b & 3.00 & bc & 6.00 & $\mathrm{c}$ & 4.00 & de \\
\hline $25 \%$ Compost + 75\% NPK & 3.3 & bc & 2.7 & abcd & 2.67 & $\mathrm{~b}$ & 2.33 & $\mathrm{~cd}$ & 7.00 & $\mathrm{~b}$ & 3.67 & e \\
\hline Zero Compost + $\mathbf{1 0 0 \%}$ NPK & 3.0 & $\mathrm{bc}$ & 2.3 & bed & 1.33 & cde & 2.00 & de & 3.33 & $\mathrm{~g}$ & 4.33 & cde \\
\hline
\end{tabular}

\section{Discussion}

The high cost of chemical fertilizers prevents most farmers from providing a balanced application of nutrients which results in lower productivity. Therefore organic composting is the most efficient feeding method for slow growing crops.

The presence of organic matter in the soil is essential in maintaining soil fertility and reducing nutrient loss. Hence, compost is a good organic fertilizer because it contains nutrients as well as organic matter. Organic matter has a number of important roles that it plays in the soil, whether in its physical structure or as a means of biological activity. In addition, the organic matter contributes greatly to improving soil properties. It provides nutrients to the soil, improves its ability to retain water, and helps the soil maintain good moisture and thus better ventilation to germinate seeds and develop plant roots (Edwards and Hailu, 2011). 
Therefore, with compost there is a much better protection from leaching compared to soluble mineral fertilizers. Especially the $\mathrm{N}$ fertilization effect of compost is limited due to low mineralization rates and microbial immobilization (Tayebeh et al., 2010).

Compost has a stimulation effect on both the microbial community in the compost substrate as well as the soil-born micro biota of soils. As mentioned by Brown and Cotton, (2011), the application of compost has increased microbial activity in comparison to the control soils. They observed microbial activity was 2.23 times greater in the compost amended soils as compared to the control soils, because organic matter found in compost provides food for microorganisms.

Better crop results were often obtained if during the first years higher amounts of compost were applied every $2^{\text {nd }}$ to $3^{\text {rd }}$ year than by applying compost in lower quantities every year (Amlinger et al., 2007). However, crop yields after pure compost application were mostly lower when compared to mineral fertilization (Agegnehu et al., 2014 and Amlinger et al., 2007), at least during the first years. This can be explained by the slow release of nutrients (especially $\mathrm{N}$ ) during mineralization of compost.

Soil organic matter has a direct effect on the availability of iron, zinc and manganese, but it has little effect on copper availability in soil (Aulakh and Mahi 2005; Chaudhury et al.,2005; Li et al. 2007). In the same way, organic fertilizers improve the supply of zinc, copper and boron (Stone and Savin 2000).

Humic substances such as humic acid, fulvic acid, are the major components (65-70\%) of soil organic matter, increase plant growth enormously due to increasing cell membrane permeability, respiration, photosynthesis, oxygen and phosphorus uptake, and supplying root cell growth (Cacco and Dell Agnolla, 1984 \& Russo and Berlyn, 1990). Humic substances are a heterogeneous mixture of naturally occurring organic materials those arise from the decay of plant and animal residues. These organic materials contain carbon, which serves as a food source for soil organisms such as bacteria, algae, fungi, and earthworms. These soil organisms break the chemical bonds in the residues as they digest the carbon. The remaining by-products serve as building blocks of humic substances, which are not easily decomposed by soil organisms (Hopkins and Stark, 2003) and this will decrease nutrients leaching with irrigation water, and so increase fertilizers use efficiency (Mikkelsen, 2005). The humic acid products are usually available in the form of inexpensive soluble salts, referred to as potassium humate (Fong et al., 2007). Potassium humate causes an increase in crop quality and tolerance of plant to drought, saline, cold, diseases and pests stresses (Gadimov et al., 2007).

Shukla et al (2009) stated that, in biological system, the micronutrient are needed in trace amounts but they are no way micro in their role, rather they play major role in enhancing efficiency of macronutrients.

On the contrary, organic manures like compost discharge nutrients very slowly to the plants and these nutrients are not directly absorb by the plants. Therefore, plants are unable access required amount of nutrients in the critical yield-forming period. Hence, an integrated approach, combining application of compost with an application of inorganic fertilizer is a good strategy for increasing crop productivity. Such combination also contributed to the improvement of physical, chemical and biological properties of soil organic matter and nutrient status. Seran et al. (2010) found that, half fold of recommended inorganic fertilizers and compost at the rate of $4 \mathrm{t} \mathrm{ha}^{-1}$ could give profitable yield and this combination could possibly reduce the cost of production in the onion cultivation. Similarly, application of half the recommended $\mathrm{N}$ and $\mathrm{P}$ rate and half the recommended rate of manure and compost as inorganic $\mathrm{N}$ equivalence resulted in yield advantages of about 129\% compared to the control (Agegnehu et al., 2014). It is clear from the results of their study that $30 \%$ of the required nitrogen fertilizer could be replaced by compost, because compost improved the use efficiency of recommended nitrogen fertilizer and reduced its cost.

The functions of effective microorganisms $\left(\mathrm{EM}_{1}\right)$ as mentioned by (Higa and Parr, 1994) were fixation of atmospheric nitrogen, decomposition of organic wastes and residues, suppression of soilborne pathogens, recycling and increased availability of plant nutrients, production of antibiotics and other bioactive compounds, production of simple organic molecules for plant uptake, complex the heavy metals to limit plant uptake, solubilization of insoluble nutrient sources and production of polysaccharides to improve soil aggregation.

In regarding to the pests infested Roselle plants and its associated natural enemies, the results revealed highly significant correlation between the infestation level of pests and each of Roselle plants height and branches numbers in cross ponding with fertilizers rates and types which activate vegetative 
growth of plants. So, the high numbers of insect pests were recorded on Roselle plants fertilized with combination of organic and inorganic fertilizer where the high vegetative attract pests and gave suit habitat to build up its population for these pests and its associated natural enemies. Hashem et al., 2017 and Shafique et al., 2016 tested the effects of organic and bio fertilizers on pests infested Roselle plants and found it decreased pests numbers when used without inorganic fertilizers. As obtained results it could be noticed that the organic and bio fertilizers can be used as integrated crop management agent in sustainable agriculture systems to get safe food with relatively low cost upon long run.

\section{References}

Abd El-Karim, A.I., M.E. El -Naggar and E.M. Amal, 2011. Survey of predaceous insects associated with four medicinal plants. J. Plant Prot. and Pathology, Mansoura Univ., 2(6): 623 - 636.

Abd El-Moneim, A.S.M. and T.E. Abd El-Wahab, 2006. Insect pests and predators in hapting Roselle plants, Hibiscus sabdariffa L. a medicinal plant in Egypt. Arch. phytopathology, Plant Protect., 39(1): $25-32$.

Abou El-Hassan, S., M. Abd Elwanis and M.Z. El-Shinawy, 2017. Application of compost and vermicompost as substitutes for mineral fertilizers to produce green beans. Egypt. J. Hort., 44 (2): 155-163a.

Afsah, A.F.E. 2005. Studies on some pests attacking medicinal and aromatic plants. Ph. D. Thesis, Fac. of Agric., Zagazig Univ.

Agegnehu, G., C. VanBeek and M. Bird, 2014. Influence of integrated soil fertility management in wheat and tef productivity and soil chemical properties in the highland tropical environment. Journal of Soil Science and Plant Nutrition, 14.

Ahmed, A.M.T. 1990. Studies on some insect pests infesting certain medicinal plants. Ph. D. Thesis, Fac. of Agric., Al-Azhar Univ., 148.

Ahmed, F., F.R. Khan and M.R. Khan, 1995. Comparative efficacy of some traditional and nontraditional insecticide against sucking insect pests of cotton. Sarhad. J. Agric., 11(6):733739.

Akanbi, W.B., A.B. Olaniyan, A.O. Togun, A.E.O. Ilupeju and O.A. Olaniran, 2009. The effect of organic and inorganic fertilizer on growth, calyx yield and quality of Roselle (Hibiscus sabdariffa L.). American-Eurasian Journal of Sustainable Agriculture, 3(4): 652-657.

Albiach, R., R.R. Canet, F. Pomares and F. Ingelmo, 2000. Microbial biomass content and enzymatic activities after the application of organic amendments to a horticultural soil. Bioresource Technology, 75: 43-48.

Amjad, A.S.A., Y.M.A. Khanif, H.A. Aminuddin, O.A. Radziah and H.A. Osumanu, 2010. Impact of potassium humate on selected chemical properties of an Acidic soil. $19^{\text {th }}$ World Congress of Soil Science, Soil Solutions for a Changing World, 1-6 August 2010, Brisbane, Australia.

Amlinger, F., S. Peyr, J. Geszti, P. Dreher, W. Karlheinz and S. Nortcliff, 2007. Beneficial effects of compost application on fertility and productivity of soils. Literature Study, Federal Ministry for Agriculture and Forestry, Envi. and Water Management, Austria. [Online] Available: www.umweltnet.at/filemanager/download/20558/ (Dec. 2013)

Aulakh, M.S. and S.S. Mahi, 2005. Interactions of nitrogen with other nutrients and water: effect on crop yield and quality, nutrient use efficiency, carbon sequestration and environmental pollution. Adv. Agron., 86: 342-409

Azarpour, E., M.K. Motamed, M. Moraditochaee and H.R. Bozorgi, 2012. Effects of bio, mineral nitrogen fertilizer management, under humic acid foliar spraying on fruit yield and several traits of eggplant (Solanum melongena L.). African Journal of Agricultural Research, 7(7): 11041109.

Bavec, F. and M. Bavec, 2006. Organic production and use of alternative crops. CRC Press. New York, USA, 242 p. [C.F., Hewidy, M., E. Sultan and M. Elsayed, 2018. Water schedule of Roselle (Hibiscus sabdariffa L.) under organic fertilization. Egypt. J. Hort., 45 (1): 53 - 64].

Baziramakenga, R. and R.R.S. Simard, 2001. Effect of deinking paper sludge compost on nutrient uptake and yields of snap bean and potatoes grown in rotation. Compost Science and Utilization, 9 (2):115-126. 
Brown, S. and M. Cotton, 2011. Changes in soil properties and carbon content following compost application: Results of on-farm sampling. Compost Science and Utilization, (2011), 19 (1) : 8897.

Cacco, G. and G. Dell Agnolla, 1984. Plant growth regulator activity of soluble humic substances. Can. J. Soil Sci., 64: 25-28.

Chang, Y.C., H.P. Huang, J.D. Hsu, S.F. Yang and C.J. Wang, 2005. Hibiscus anthocyanins rich extract-induced apoptosis cell death in human promyelocytic leukaemia cells. Toxicol. Appl. Pharmacol., 205(3): 201-212.

Chaudhury, J., U.K. Mandal, K.L. Sharma et al., 2005. Assessing soil quality under long- term ricebased cropping systems. Commun. Soil Sci. Plant Anal., 36:1-21. [C.F., Ibrahim, H. A. K. 2019. Effect of foliar application of compost water extract, humic acid, EDTA and micronutrients on the growth of fenugreek plants under sandy soil condition. International Journal of Environmental Science and Technology, (2019) 16: 7799-7804].

Daly, M.J. and D.P.C. Stewart, 1999. Influence of "Effective Microorganisms" (EM) on vegetable production and carbon mineralization - a preliminary investigation. Journal of Sustainable Agriculture, 14: $15-25$.

Dalziel, J.M., 1973. The useful plants of west Tropical Africa. The Crown Agents, London), 314-315.

Du C.T. and F.J. Francis, 1973. Anthocyanin of Roselle. J. Food Sci., 38 (5): 810-820.

Duncan, D.B. 1958. Multiple Ranges and Multiple F-test Biometric.11: 1-24.

Edwards, S. and A. Hailu 2011. How to make compost and use. In: Ching, L.L., Edwards S. and Nadia H.S. (Eds), Climate Change and Food Systems Resilience in Sub-Sarahan Africa. FAO, Italy, 379- 436.

El-Sayed, A.M.K., F.A. Abdel-Galil, Y.A. Darwish and G.H. Abou El-Hagag, 1990. Incidence and dominance of arthropods associated with Roselle, caraway and coriander plants in upper Egypt. Assiut J. of Agric. Sci., 21(3): 61-75.

El-Sherif, A.M.A., 2016. Sesame (Sesamum indicum L.) yield and yield components influenced by nitrogen and foliar micronutrient applications in the Fayoum region, Egypt. Egypt. J. Agron., 38 ( 3): 355-367.

Epstien, E. 1972. Mineral nutrition of plants. Principles and perspectives."John Wiley and Sons. Inc. USA.

Esmaeili, A., A. Najarzadeh, R. Hemayati, J.Z. Reza and M. Taghizadeh, 2014. Effects of Hibiscus subdariffa on kidney function, albuminuria, total antioxidant capacity, hypertension and HSCRP in patients with diabetic nephropathy. Nutrition and Food Sciences Research, 1 (1); 106107.

Fong, S.S., L. Seng and H.B. Mat, 2007. Reuse of Nitric acid in the oxidative pretreatment step for preparation of humic acids from low rank coal of Mukah. Sarawak. J. Braz. Chem. Soc., 18(1): 41-46.

Fuleki, T. and F.J. Francis, 1968. Quantitative methods of anthocyanin 1. Extraction and determination of anthocyanin in cranberries. J. Food Sci., 33(1):72.

Gadimov, A., N. Ahmaedova and R.C. Alieva, 2007. Symbiosis nodules bacteria Rhizobium leguminosarum with Peas (Pisum sativum) nitrate reductase, salinification and potassium humus. Azarbayjan National Academy of Sciences. International Humic Substances Society. What are humic substances? Focus on form: Retrieved May 27, 2009, from http://ihss.gatech.edu/ihss2/whatarehs.html.

Gomez, K.A. and A.A. Gomez, 1984. Statistical Procedures for Agricultural Research. John Wiley \& Sons Inc., Singapore, 680.

Hammad, K.A.A. and A.M. Mohsen, 2000. . Insects infesting Roselle and their associated natural enemies in newly reclaimed sandy land. Egypt. J. Appl. Sci., 15 (7): 618B642.

Hanafy, M.S., 2009. Effect of planting distances and some microelements on the growth, yield and some chemical constituents of Roselle (Hibiscus sabdariffa, L.) plant. J. Product. \& Dev., 14(3): $545-570$.

Hashem, A.E.A. Hanan, Abeer, E.A. EL-Hadidy and E.A. Ali, 2017. Impact of some safe agricultural treatments on insect pests, vascular wilt disease management and Roselle (Hibiscus sabdariffa L.) productivity under Siwa Oasis condition. Int. J. Environ., 6 (4): 139-162. 
Hewidy, M., E. Sultan and M. Elsayed, 2018. Water schedule of Roselle (Hibiscus sabdariffa L.) under organic fertilization. Egypt. J. Hort., 45 (1): $53-64$.

Higa, T., 2002. Eine Revolution zur Rettung der Erde: mit Effektiven Mikroorganismen (EM) die Probleme unserer Welt Lösen. 3. Aufl.- OLV Organischer Landbau - Verlag: Xanten, Germany.

Higa, T. and J.F. Parr, 1994. Beneficial and Effective Microorganisms for a sustainable agriculture and environment. International Nature Farming Research Center, Atami, Japan.

Hopkins, B. and J. Stark, 2003. Humic acid effects on potato response to phosphorus. Presented at the Idaho Potato Conference, January, 22-23.

Hu, C. and Y. Qi, 2013. Long-term Effective Microorganisms application promote growth and increase yields and nutrition of wheat in China. European J. of Agronomy, 46: 63-67.

Hussein, M. S., S. E. El-Sherbeny, H.M. El-Saeid and M.M. Kandeel, 1989. Field experiments of foliar application with B-9 and micronutrients on Hibiscus sabdariffa L. Egyptian Journal of Horticulture, 1989, publ. 1990, 16(1): 59-68; 31 ref.

Ismail, O.M.N., 2001. Studies on some insects attacking aromatic and medicinal plants. M.Sc. Thesis, Fac. Agric., Cairo Univ., Egypt.

Jackson, M.L., 1967. Soil Chemical Analysis. Prentice Hall of India, Private Limited; New Delhi, 115.

Khalil, S.E. and R.M.M. Yousef, 2014. Study the effect of irrigation water regime and fertilizers on growth, yield and some fruit quality of Hibiscus sabdariffa L. International Journal of Advanced Research, 2(5): 738-750.

Khattab, M.E., 1997. Growth and yield response of Roselle new cultivars to foliar nutrient application. Bulletin of the National Research Centre, Cairo, 22(4): 473-494; 21 ref.

Khosravi, H.M., B.A.J. Khanabadi, M.A. Ardekani, F. Fatehi and M.N. Shadkam, 2009. The effects of sour tea (Hibiscus sabdariffa) on hypertension in patients with type II diabetes. Journal of Human Hypertension, 23: 48-54.

Li, B.Y., D.M. Zhou, L. Cang et al., 2007. Soil micronutrient availability to crops as affected by longterm inorganic and organic fertilizer applications. Soil Tillage Res., 96:166-173. [C.F., Ibrahim, H.A.K. 2019. Effect of foliar application of compost water extract, humic acid, EDTA and micronutrients on the growth of fenugreek plants under sandy soil condition. International Journal of Environmental Science and Technology, (2019) 16: 7799-7804].

Mahmoud, E.K., N. Abd EL-Kader, P. Robin, N. Akkal-Corfini and L. Abd El-Rahman, 2009. Effects of different organic and inorganic fertilizers on cucumber yield and some soil properties. World J. Agric. Sci., 5 (4): 408-414.

Maunde, S.M. and L. Aliyu, 2012. Effects of nitrogen, phosphorus and farmyard manure on growth and nutrient uptake by Roselle (Hibiscus sabdariffa L.) in the Nigerian savanna. Nigeria Journal of Soil and Environmental Research, 10: $42-49$.

Mazumder, B.C. and K. Majumder, 2003. Methods of Physiological Analysis of Fruits. Daya Published House, Delhi, India.

Mesbah, H.A., K.S. Moursii, I.A. Abou El-Fadl and E.A. Zakzouk, 1982. some pests infesting medicinal and aromatic plants in Egypt. II- Pests found on Roselle (Hibiscus sabdariffa L.) in Alexandria district. Proc. of Egypt National Conf. Entomol., 12(1): 297-309.

Mikkelsen, R.L. 2005. Humic Materials for Agriculture. Better Crops, 89.

Mortvedt, J., F. Cox, L. Shuman and R. Welch, 1991. Micronutrients in Agriculture." $2^{\text {nd }}$ Ed. Published by Soil Soc. Amer. Inc. Madison, Wisconsin, USA, 760.

Osman, D.F., Heba A. Ismail, I.M. Kelany, Shadia M. Omara and Sh.M. Abd-Allah, 2017. Survey of main insect species associated with certain medicinal and aromatic plants at Awlad-Sakr district, Sharkia Governorate. Zagazig J. Agric. Res., 44 (5): 1839-1850.

Rashad, S.E., 1976. Experience with pollination of tropical crops. Apiculture in tropical climate, London, 109-112.

Rashad, S.E., 1978. Utilization of non Apis bees as crop pollinators. EG. ARS, 66:48.

Russo, R.O. and G.P. Berlyn, 1990. The use of organic biostimulants to help low input sustainable agriculture. J. Sustain. Agric., 1: 19-42.

Seran, T.H., S. Srikrishnah and M.M.Z. Ahamed, 2010. Effect of different levels of inorganic fertilizers and compost as basal application on the growth and yield of onion (Allium cepa L.). The Journal of Agricultural Sciences, 5 (2): 64-70. 
Shafique, H.A., V. Sultan, S. Ehteshamul-Haque and M. Ather, 2016. Management of soil-borne diseases of organic vegetables. Journal of Plant production Research, 1, 56 (3): 222- 230.

Shukla, A.K., B.S. Dwivedi, V.K. Singh and M.S. Gill, 2009. Macro role of micronutrients. Indian Journal of Fertilisers, 5 ( 5): 11-12.

Stone, J.P. and R. Savin, 2000. Grain quality and its physiological determinants in wheat ecology and physiology of wheat determinants. Viva Books Private Limited, New Delhi, 85-96. [C.F., Ibrahim, H. A. K. 2019. Effect of foliar application of compost water extract, humic acid, EDTA and micronutrients on the growth of fenugreek plants under sandy soil condition. International Journal of Environmental Science and Technology, (2019) 16: 7799-7804].

Tayebeh, A., A. Abass and A.K. Seyed, 2010. Effect of organic and inorganic fertilizers on grain yield and protein banding pattern of wheat. Australian Journal of Crop Science (AJCS), 4(6):384389.

Tsai, P.J., J. McIntosh, P. Pearce, B. Camden and B.R. Jordan, 2002. Anthocyanin and antioxidant capacity in Roselle (Hibiscus Sabdariffa L.) extract. Food Research International, 35(4):351356

Wang, M.L., B. Morris, B. Tonnis, J. Davis and G.A. Pederson, 2012. Assessment of oil content and fatty acid composition variability in two economically important Hibiscus species. Journal of Agricultural and Food Chemistry, 60 (26)): 6620-6626. 